\title{
Extraordinary Transmission Through Arrays of Slits: A Circuit Theory Model
}

\author{
Francisco Medina, Fellow, IEEE, Francisco Mesa, Member, IEEE, and Diana C. Skigin, Member, IEEE
}

\begin{abstract}
Extraordinary transmission and other interesting related phenomena for 1-D periodic arrays of slits (compound diffraction gratings) have recently been the object of intense research in the optics and solid state physics communities. This case should be differentiated from the extraordinary transmission through arrays of small apertures on metal screens since small holes only support below-cutoff modes, whereas slits can also support transverse electromagnetic modes without cutoff frequency. In this paper, an equivalent-circuit approach is proposed to account for the most relevant details of the behavior of slit-based periodic structures: extraordinary transmission peaks, Fabry-Pérot resonances, and transmission dips observed in compound structures. The proposed equivalent-circuit model, based on well-established concepts of waveguide and circuit theory, provides a simple and accurate description of the phenomenon that is appropriate for educational purposes, as well as for the design of potential devices based on the behavior of the structures under study.
\end{abstract}

Index Terms-Extraordinary transmission, diffraction gratings, impedance matching, surface plasmon polaritons (SPPs).

\section{INTRODUCTION}

$\mathbf{T}$ HE theoretical and experimental study of extraordinary optical transmission through periodic arrays of electrically small apertures in a metal screen has been a very popular research topic since the phenomenon was first reported ten years ago [1]. The reader can find accurate descriptions of the phenomenon and the state of the art of the research on it in a couple of good and relatively recent review papers [2], [3]. Enhanced transmission has also been reported for 2-D quasi-periodic distributions of subwavelength apertures [4]. Another rel-

Manuscript received February 24, 2009; revised July 16, 2009. First published December 11, 2009; current version published January 13, 2010. This work was supported by the Spanish Ministerio de Educación y Ciencia and European Union FEDER funds under Project TEC2007-65376 and Consolider-Ingenio 2010 "Engineering Metamaterials" Project CSD2008-00066, and by Junta de Andalucía under Project TIC-253. The work of D. C. Skigin was supported in part by the Consejo Nacional de Investigaciones Científicas y Técnicas under CONICET Grant PIP 5700, by the Universidad de Buenos Aires under UBA Grant X283 and UBA Grant X208, and by the Agencia Nacional de Promoción Científica y Tecnológica under ANPCYT-BID Grant 802/OC-AR03-14099.

F. Medina is with the Microwaves Group, Department of Electronics and Electromagnetism, Faculty of Physics, University of Seville, 41012-Seville, Spain (e-mail:medina@us.es).

F. Mesa is with the Microwaves Group, Department of Applied Physics 1, ETS de Ingeniería Informatica, University of Seville, 41012-Seville, Spain (e-mail:mesa@us.es).

D. C. Skigin is with the Applied Electromagnetics Group, Physics Department, University of Buenos Aires, Ciudad Universitaria, C1428EHA Buenos Aires, Argentina, and also with the Consejo Nacional de Investigaciones Científicas y Técnicas (CONICET), C1033AAJ, Buenos Aires, Argentina (e-mail:dcs@df.uba.ar).

Digital Object Identifier 10.1109/TMTT.2009.2036341 evant situation corresponds to enhanced transmission through single apertures around which the surface of the screen is periodically structured with corrugations (see, e.g., [5]-[8] or [3]). Transmission through holes that are not electrically small has also been object of attention ([3], [9], and [10] among many others), but this case is closer to the study of the frequency-dependent radar cross section of objects whose size is on the order of the wavelength [11], [12].

The original experiments [1] and most of the published literature [2], [3] deal with the transmission through 2-D arrays of small holes. Surface plasmon polaritons (SPPs) excited on the periodically structured surface of the metal screen can explain the transmission peaks observed in the experiments. SPP-Bloch waves of this type-also called spoof plasmons, as those theoretically studied in [13] and [14] and experimentally demonstrated in [15] and [16] — would explain the phenomenon both at optical and microwave/millimeter-wave [17] frequencies (note that metals behave as plasmas at optical frequencies, but rather as perfect conductors at microwave frequencies). Surface waves supported by periodically structured surfaces (mostly in the 1-D case) are well known in the microwave field theory discipline [18], and they have also been studied in depth in the optical regime [19]. Although the explanation of extraordinary transmission in terms of the excitation of surface waves at both sides of the perforated screen gives an adequate account of the phenomenon, some alternative theories suggest that this approach could be incomplete [20]-[22]. Parallel to this controversy, a different point of view has recently been proposed by some of the authors of this paper. In this new approach, an equivalent-circuit model based on the concept of perfect impedance matching explains all the details of the observed/calculated transmission spectra in the case of 2-D arrays of holes [23], [24] with a high degree of accuracy for typical geometries reported in the literature. This model is based on standard concepts of waveguide theory and gives many easily-derived qualitative and quantitative predictions about the behavior of perforated screens. Moreover, the model predicts extraordinary transmission in some systems where surface plasmons and periodicity are absent [24]. Simple equivalent circuits account for the essential details of transmission spectra on the basis of a central idea: impedance matching is required for perfect or almost perfect transmission and this is possible in different structures thanks to the frequency-dependent behavior of the electrical parameters of the higher order TM modes. A similar point of view has been independently developed by Gordon et al. [25], [26] (for infinitesimally thin screens and without circuit model). Another approach that is close to the classical theory of waveguides and resonators, as well as frequency-selective surfaces can be found in an excellent paper by Kirilenko and Perov [27], which was preceded by 
[28]. The set of papers [24], [25], [27] defines a different paradigm (based on the concept of impedance matching) for the explanation of extraordinary transmission that avoids the possible controversies associated with SPP Bloch-like theories.

However, the topic of this paper is not transmission through 2-D periodic structures, but through 1-D diffraction gratings. It is worth to mention that some of the first theoretical explanations of extraordinary transmission considered a simplified situation where the 2-D array of circular holes was substituted by a 1-D array of infinitely long slits [30]. The results reported in [30] and subsequent papers [20], [31] show that large transmission peaks occur at wavelengths close to the period of the 1-D periodic grating provided the screen was not infinitesimally thin. In contrast to 2-D structures, the transmission peak disappears as the screen thickness of the slit-based structure tends to zero. A single isolated peak is also predicted for 1-D nonzero thickness gratings instead of the couple of closely spaced peaks obtained for 2-D arrays of holes in thick screens [2]. Nevertheless, what is radically different from the 2-D case is the existence of Fabry-Pérot-like resonances in the case of electrically thick screens (around half-wavelength or more) [31], [32]. These resonances are not related to the periodicity, but to the thickness of the screen. They are absent in 2-D arrays of small holes (thick screen case) because the modes inside the holes are evanescent. However, propagating TEM waves are always present inside the slits. Hence, Fabry-Pérot-like resonances are possible even for a single slit, a rather expected fact that has been studied again in the frame of extraordinary transmission research [33]-[35]. In addition to the appearance of Fabry-Pérot resonances, another interesting phenomenon arises when compound gratings (slits systems with several slits per period) are analyzed. In this case, phase resonances have been theoretically predicted [32], [36], [37] and experimentally observed [38]-[40]. These resonances give place to very narrow transmission zeros (dips) allocated near the center of relatively wide Fabry-Pérot transmission bands (making the structures behave as highly selective notch filters). It has been brought to the authors' attention [41] that the physics of electrically thin and thick 1-D gratings was already dealt with by former Soviet Union scientists [42]-[44] in the 1960s/1970s and that the published results covered the essential contributions reported in the most recent Western literature on the topic.

It is our purpose in this paper to adapt the analysis method and concepts introduced in [24] (and, to a some extent, in [25] and [27]) to account for the above-mentioned transmission phenomena of 1-D simple/compound gratings. A first attempt of using the methodology in [24] to deal with 1-D gratings was reported in [29]. Now the ideas just sketched in [29] will be explained in detail, and a number of extensions and improvements will be introduced. More specifically, the equivalent-circuit model of simple diffraction grating is improved, a new equivalent circuit is introduced for compound gratings, and losses will also be incorporated in the circuit model. One of the relevant features of the proposed approach is that the circuit model is very useful for design purposes and, of course, gives a different perspective on the physical phenomenon. Moreover, it provides a unified framework for extraordinary transmission both in 1-D and 2-D periodic structures.

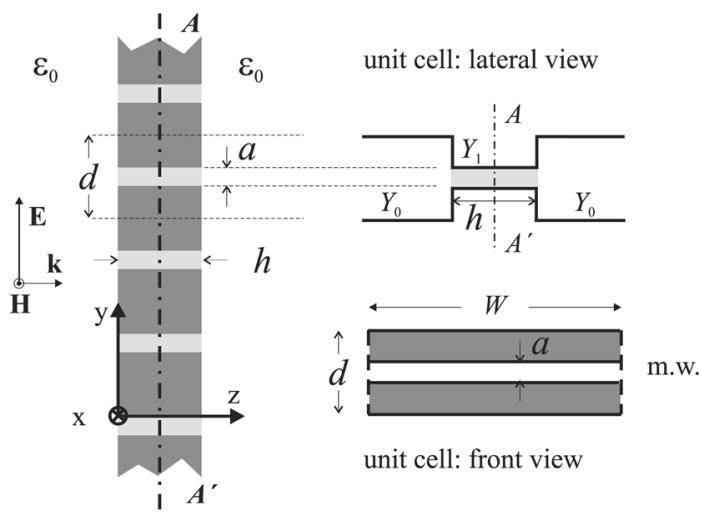

(a)

(b)

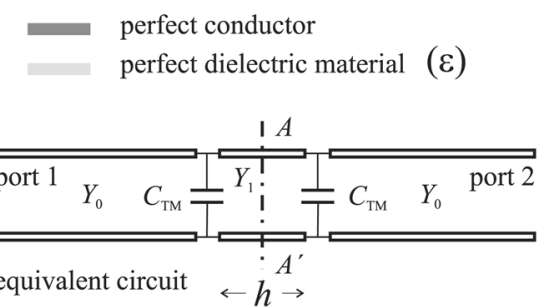

(c)

Fig. 1. (a) Lateral view of a perfect conducting screen with periodic array of slits. Dimensions: period $=d$, screen thickness $=h$, slit width $=a$. (b) Longitudinal and transversal views of the unit cell for normal incidence. The width, $W$, of the unit cell is arbitrary. (c) Equivalent circuit of the discontinuity problem. e.w. denotes electric wall, m.w. denotes magnetic wall.

\section{Transmission Through Simple GratingS}

The simplest situation will be considered first: an infinite periodic array of narrow and infinitely long slits made on a perfect metal screen of finite thickness $h$. The lateral view of this structure is shown in Fig. 1(a). The slits may be filled with a dielectric of permittivity $\varepsilon=\varepsilon_{r} \varepsilon_{0}$. A TEM plane wave with the electric field polarized along the direction perpendicular to the slits and parallel to the screen surface ( $y$-direction) impinges on the structure. Due to the periodic nature of the structure along the $y$-direction and the assumed excitation, it is apparent that only the unit cell shown in Fig. 1(b) requires our attention (this figure shows the longitudinal and transversal views of the unit cell). Thus, the original problem is reduced to the computation of the scattering parameters of a TEM mode supported by a parallel-plate transmission line (the one having characteristic admittance $Y_{0}=(W / d) / \eta_{0}$ in Fig. 1(b), with $\eta_{0}=$ $\sqrt{\mu_{0} / \varepsilon_{0}}$ being the characteristic impedance of TEM waves in free space) when a finite section of length $h$ of another parallel-plate transmission line is inserted [the line having characteristic admittance $Y_{1}=\sqrt{\varepsilon_{r}}(W / a) / \eta_{0}$ in Fig. 1(b)]. Note that the width $(W)$ of the parallel-plate transmission line can be arbitrarily chosen since no variations of the fields along the $x$-direction are allowed. In this situation, only TEM and TM (transverse magnetic) modes (with respect to the propagation direction, $z$ ) can be excited at the discontinuities (here, and due to the symmetry of the problem under study, only even TM modes will be considered). As is well known [18], the influence of below cutoff TM modes can be accounted for by an equivalent capacitance, which leads to the equivalent circuit shown in 
Fig. 1(c). In this circuit, the capacitance $C_{\mathrm{TM}}$ can be considered almost frequency independent as long as the working frequency is far from the cutoff frequency of the TM modes supported by the transmission line with characteristic admittance $Y_{0}$. It will correspond to a frequency regime below the onset of the first grating lobe; namely, below the onset frequency of the first TM mode (the $\mathrm{TM}_{02}$ mode). This specific range will be denoted as Fabry-Pérot regime and will be considered in Section II-A.

\section{A. Fabry-Pérot Regime}

The Fabry-Pérot regime corresponds to frequencies well below the cutoff frequency of the first TM mode of the external waveguide. Due to the symmetry of the equivalent circuit in Fig. 1(c), the application of even/odd excitation analysis easily leads to the following scattering parameters.

1) Even (e) Excitation: In this case, the plane $A A^{\prime}$ depicted in Fig. 1 is a magnetic wall. Therefore, the reflection coefficient for even excitation, $S_{11}^{e}$, is given by

$$
S_{11}^{e}=\frac{Y_{0}-\mathrm{j} \omega C_{\mathrm{TM}}-\mathrm{j} Y_{1} \tan (\theta / 2)}{Y_{0}+\mathrm{j} \omega C_{\mathrm{TM}}+\mathrm{j} Y_{1} \tan (\theta / 2)} .
$$

2) Odd (o) Excitation: In this case, the plane $A A^{\prime}$ depicted in Fig. 1 is an electric wall, and the reflection coefficient for odd excitation, $S_{11}^{o}$, is given by

$$
S_{11}^{o}=\frac{Y_{0}-\mathrm{j} \omega C_{\mathrm{TM}}+\mathrm{j} Y_{1} \cot (\theta / 2)}{Y_{0}+\mathrm{j} \omega C_{\mathrm{TM}}-\mathrm{j} Y_{1} \cot (\theta / 2)} .
$$

In the above expressions, $\theta$ is the electrical length of the transmission line section of physical length $h(\theta=k h$ with $k$ being the wavenumber of the dielectric inside the slits).

Now applying the superposition principle, the scattering parameters for the structure under study can be written as

$$
S_{11}=\frac{1}{2}\left[S_{11}^{e}+S_{11}^{o}\right] \quad S_{12}=\frac{1}{2}\left[S_{11}^{e}-S_{11}^{o}\right] .
$$

(Papers published in optics and other physics journals usually employ power-referred quantities such as reflectivity and transmissivity; the reflection coefficient is then $\left|S_{11}\right|^{2}$ and the transmission coefficient is $\left|S_{12}\right|^{2}$.) Obviously, total transmission is obtained for those frequencies at which $\left|S_{11}\right|=0$. This total transmission has been called extraordinary transmission in the literature on the subject. ${ }^{1}$

The condition for total transmission derived from the circuit model in Fig. 1(c) is then given by the following equation:

$$
\tan \theta=-\frac{2 Y_{1} \omega C_{\mathrm{TM}}}{Y_{1}^{2}-Y_{0}^{2}-\left(\omega C_{\mathrm{TM}}\right)^{2}} .
$$

The condition (4) corresponds to Fabry-Pérot resonances of the transmission line section of length $h$ with characteristic admittance $Y_{1}$ and loaded at both ends with the capacitance $C_{\mathrm{TM}}$.

\footnotetext{
${ }^{1}$ The term "extraordinary" seems to be commonly used because the slit width $a$ is much smaller than the corresponding wavelength. However, it must be recognized that, although the subwavelength size of the holes is important in the case of 2-D periodic arrays of holes, the presence of subwavelength slits is not an issue for transmission because TEM modes are allowed inside the slits at any wavelength. Due to this reason, we will not follow this common practice and, instead, "total transmission" will be used for the transmission associated with Fabry-Pérot resonances. "Extraordinary transmission" will be reserved for the transmission peak located near the onset of the first grating lobe.
}

These resonances are observed if the screen is electrically thick (approximately half wavelength or more). The effect of $C_{\mathrm{TM}}$ is just to shift the resonance peaks to frequencies lower than those expected if the edge effects were neglected $\left(C_{\mathrm{TM}}=0\right.$; in such a case, transmission peaks would be obtained for values of $\theta$ exactly equal to integer multiples of $\pi$ ). In the frequency range considered in this section (far from the onset of the first grating lobe), the typical values of $C_{\mathrm{TM}}$ means that condition (4) can always be satisfied for values of $\theta$ not far, but always below $n \pi(n=1,2, \ldots)$. Since $C_{\mathrm{TM}}$ is almost frequency independent in this regime, its value can be closely estimated from a purely electrostatic analysis $\left(C_{\mathrm{TM}}\right.$ can be obtained from the solution of a 2-D Laplace problem in the $y z$-plane). Once $C_{\mathrm{TM}}$ is known, a simple Newton-Raphson scheme could be used to obtain the solutions of (4) (a graphical method would provide more physical insight about the nature of the expected solutions).

The capacitance $C_{\mathrm{TM}}$ can alternatively be viewed as the edge capacitance of the parallel-plate capacitor formed by the two conductors defining the slit region. Provided that $Y_{1} \gg Y_{0}$ (as usually happens in the slit problems treated in the literature, where $a \ll d$ ) and that $h / a$ is not too small, it is possible to interpret the result in (4) saying that the transmission line of characteristic admittance $Y_{1}$ has an equivalent length given by its actual physical length $h$, plus an excess length associated with the edge capacitances $\Delta h=2 c C_{\mathrm{TM}} / Y_{1}$, where $c$ is the speed of light in the dielectric inside the slits. The use of equivalent lengths is a common practice in microwave engineering. Following this rationale, the total transmission frequencies $f_{\mathrm{TT}}$ would be given by

$$
f_{\mathrm{TT}}=\frac{n}{2} \frac{c}{h+\Delta h} .
$$

The above point of view is consistent with the results reported in [34] for a single slit made on a thick aluminum screen. $\Delta h$ in (5) perfectly accounts for the wavelength shift (with respect the theoretical value of the Fabry-Pérot wavelength) studied in [34].

\section{B. Extraordinary Transmission Regime}

In the case of periodic gratings (but not for single slits), there is still another possibility to satisfy (4), which is not related to Fabry-Pérot resonances. In the frame of our model, this possibility comes from the fact that $C_{\mathrm{TM}}$ strongly depends on frequency when the free-space wavelength is close to the period $d$ of the periodic system (note that $C_{\mathrm{TM}}$ hardly changes with frequency in the simple case of a single slit treated in [33]-[35]). This strong frequency dependence can readily be explained from conventional waveguide theory. The frequency $f_{\mathrm{TM} 2}^{c}=c_{0} / d$ (with $c_{0}$ being the speed of light in free space) will denote the cutoff frequency of the first TM mode supported by the parallel-plate transmission line with characteristic admittance $Y_{0}$ (namely, the $\mathrm{TM}_{02}$ mode). This frequency also corresponds to the onset of the first grating lobe of the diffraction grating. As is well known [18], the input admittance associated with a TM mode below cutoff is imaginary and becomes singular at its corresponding cutoff frequency. This behavior can be incorporated in the equivalent circuit of Fig. 1(c) by means of an equivalent capacitance $C_{\mathrm{TM}}(f)$ with 
the following singular behavior reported in [24, eqs. (2) and (3)]:

$$
C_{\mathrm{TM}}(f)=C_{0}+\frac{A_{\mathrm{TM}_{02}}}{2 \pi f \eta_{0} \sqrt{\left(\frac{f_{\mathrm{TM}_{02}}^{c}}{f}\right)^{2}-1}} .
$$

The (almost) frequency-independent capacitance $C_{0}$ accounts for all the higher order TM modes, and the frequency-dependent contribution is associated with the dominant TM mode of the external waveguide. The coefficient $A_{\mathrm{TM}_{02}}$ mainly depends on the ratio $a / d$ and can be estimated from a mode-matching analysis using very few modes. Note that $C_{\mathrm{TM}}(f)$ becomes infinite at $f=f_{\mathrm{TM}_{02}}^{c}$, which means that our circuit model predicts total reflection (with phase $\pi$ ) at that frequency. This transmission zero is the so-called Wood-Rayleigh anomaly, a phenomenon well known in the areas of diffraction gratings and/or frequency-selective surfaces. It must be mentioned here that zero transmission should not be expected for wide slits in the same way that the edge capacitance in our circuit model would not exactly account for the actual electromagnetic problem (more sophisticated circuit models should then be used, following the guidelines reported in [45] and [46]). Anyhow, for not too wide slits, (almost) zero transmission Wood-Rayleigh anomalies will occur at the onset of any TM mode (the first two zero transmission Wood anomalies can be clearly observed in the results reported in [42] and [43]). It is worth mentioning that the onset of a new TM mode in this model is equivalent to the onset of a new grating lobe when the problem is viewed from the diffraction theory perspective. Coming back to our extraordinary transmission problem, but considering now that $h \ll d$, it is found that for frequencies around $f_{\mathrm{TM}_{02}}^{c}$ no Fabry-Pérot resonances are allowed (these would occur at much higher frequencies). If $h \ll d$, some useful approximations can be done in (1) and (2) (apart from the obvious $\tan \theta \approx \theta$ ). Denoting $C_{\mathrm{pul}}=\varepsilon W / a$ and $L_{\mathrm{pul}}=\mu_{0} a / W$ the per unit length (pul) capacitance and inductance of the transmission line of characteristic admittance $Y_{1}$, respectively, and defining $C_{\mathrm{eq}}=C_{\text {pul }} h / 2$ and $L_{\mathrm{eq}}=L_{\mathrm{pul}} h / 2$ as the quasi-static capacitance and inductance of the short section of transmission line of length $h / 2$ associated with the slit region, respectively, $t$ can be written that $\mathrm{j} Y_{1} \tan (\theta / 2) \approx \mathrm{j} \omega C_{\text {eq }}$ and $\mathrm{j} Y_{1} \cot (\theta / 2) \approx 1 /\left(\mathrm{j} \omega L_{\text {eq }}\right)$. These approximations simply account for a lumped-element modeling of the transmission line section in Fig. 1(c) (very short in the present case). Under these simplifications, condition (4) for total transmission can be written as

$$
L_{\mathrm{eq}} Y_{0}^{2}=\left(C_{\mathrm{TM}_{02}}+C_{\mathrm{eq}}\right)\left(1-\omega^{2} L_{\mathrm{eq}} C_{\mathrm{TM}_{02}}\right) .
$$

It can be checked that (7) is always satisfied for a frequency below $f_{\mathrm{TM}_{02}}^{c}$ as long as $L_{\text {eq }} \neq 0$. As stated before, the reason is that $C_{\mathrm{TM}_{02}}$ will monotonically increase up to infinity at that frequency in such a way that all the possible values of $C_{\mathrm{TM}_{02}}$ are swept before reaching $f_{\mathrm{TM}_{02}}^{c}$. Thus, there is always a single frequency value for which condition (7) is satisfied. Note that the total transmission frequency is usually very close to $f_{\mathrm{TM}_{02}}^{c}$ and that this frequency is not related to the thickness of the screen [as in (5)], but to the period $d$ of the structure. For the above reasons, the associated total transmission phenomenon is what actually deserves to be called extraordinary transmission in the 1 -D grating case [30]. It is worth mentioning here the resemblance of this reasoning with the theory reported in [31], where a different language is used but the same physics is described. As previously mentioned, similar results were obtained more than 40 years ago using different methods [42], [43].

It is important to notice that our approach predicts a single transmission peak (instead of the double peak appearing in 2-D structures with thick screens), which is fully consistent with full-wave numerical simulations and experiments. On the other hand, (7) predicts the absence of the transmission peak if $L_{\mathrm{eq}}=0\left(C_{\mathrm{TM}_{\mathrm{O} 2}}\right.$ cannot take negative values). This means that zero thickness metallic gratings cannot exhibit extraordinary transmission peaks, in contrast with 2-D arrays of holes in zero thickness screens. This qualitative prediction is again in perfect agreement with experience and full-wave numerical data. The different behavior for zero-thickness screen between 1-D and 2-D gratings is explained by the absence of evanescent TE (transverse electric) modes excited by the discontinuity analyzed in this paper (a capacitive iris in the terminology of microwave engineers). In the 2-D case, both TM and TE modes are scattered by the diaphragm in such a way that a nonzero inductance is always provided by the below-cutoff TE modes of the external waveguide even if the thickness of the screen is zero (i.e., even if there is no inductance associated with the inner hole).

\section{Numerical Results}

In this section, the quantitative predictions of our model will be compared with numerical data obtained via a full-wave mode-matching scheme. The value of the capacitance $C_{\mathrm{TM}}$ in the model is obtained using a Laplace's solver and its frequency dependence near the onset of the first TM mode is estimated from a few low-frequency values of the scattering parameters (as was done in [24]). These values are obtained using the mode-matching method reported in [32]. Alternatively, the relative weight of the $\mathrm{TM}_{02}$ mode in the evanescent spectrum of the fields scattered by the slit [i.e., the value of $A_{\mathrm{TM}_{02}}$ in (6)] can be estimated from a low-order mode matching.

Fig. 2 plots the transmittance through simple arrays of parallel slits for two sets of geometrical parameters. The results obtained with the rigorous numerical method employed in [32] and with the proposed circuit model agree very well in the whole band. This figure shows two relatively wide transmission peaks associated with inner Fabry-Pérot resonances. These peaks are accurately computed without considering the frequency dependence of $C_{\mathrm{TM}}$, but another narrow peak can be observed very close to the Wood's anomaly $(\lambda / d=1)$. The almost perfect agreement between the numerical and circuit model results has been verified for many sets of geometrical parameters. It can then be concluded that our simple proposed model catches all the essential features of the phenomenon, which clearly proves the validity of our approach for 1-D gratings (as it was already proved for 2-D perforated screens [24]). Moreover, this good matching is carried out without resorting to either surface plasmons or the plasmonic behavior of metals at optical frequencies. 




Fig. 2. Comparison of the transmittance obtained with full-wave simulation following the method in [32] (solid lines) and the circuit model in this work (dots). Dimensions are $d=5 \mathrm{~mm}$ and $h=6 \mathrm{~mm}$ with $a=2 \mathrm{~mm}$ or $a=0.5 \mathrm{~mm}$ (see Fig. 1 for notation). Using $W=1 \mathrm{~m}$ (arbitrarily chosen), the values of the electrical parameters used in circuit simulation are: (top) $Y_{0}=0.531 \Omega^{-1}, Y_{1}=1.327 \Omega^{-1}, C_{\mathrm{TM}}=1.586 \mathrm{pF}$ and (bottom) $Y_{0}=0.53 \Omega^{-1}, Y_{1}=5.308 \Omega^{-1}, C_{\mathrm{TM}}=5.285 \mathrm{pF}$. The values of $C_{\mathrm{TM}}$ are valid, except near $f_{\mathrm{TM}_{02}}^{c}$, where the frequency-dependent behavior in (6) must be considered.

\section{TRANSMISSION Through COMPOUND GRATINGS}

In addition to the study of simple gratings, increasing attention has recently been paid to the analysis of more complex geometries, the so-called compound gratings. These structures are 1-D periodic gratings having more than one slit per unit cell. The compound gratings exhibit interesting transmission properties that have been studied in depth, first via full-wave rigorous numerical methods [32], [36], [37] and later experimentally [38]-[40]. A first effect reported in [32] was that the addition of slits to the period leads to a widening of the transmission maxima (when compared to the single slit case), but a second more interesting phenomenon that is also found is the appearance of sharp dips in the transmission response associated with certain phase resonances. This kind of transmission dips were reported in [44] 30 years ago in a similar system and some of the authors of this paper have reported on a microstrip circuit exhibiting similar transmission spectra [47]. From the results in [32], it is clear that the number of dips is related to the number of slits per period. For normal incidence, at least three slits per period are required to observe transmission dips. For three or four slits per period, a single dip is obtained for every Fabry-Pérot transmission band. Two dips are possible when five or six slits per period are present. The number of dips per transmission band increases, following this rule, as the number of slits per period increases.

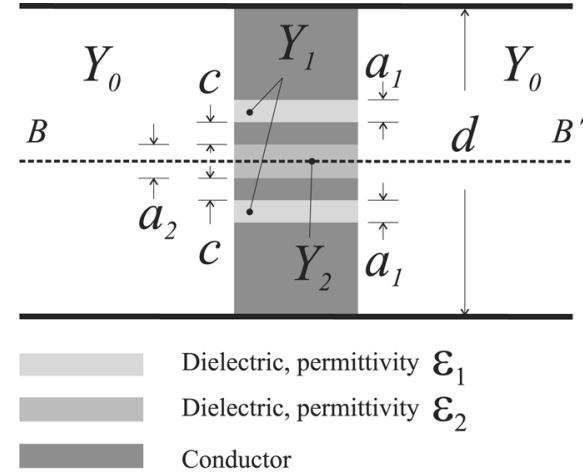

(a)

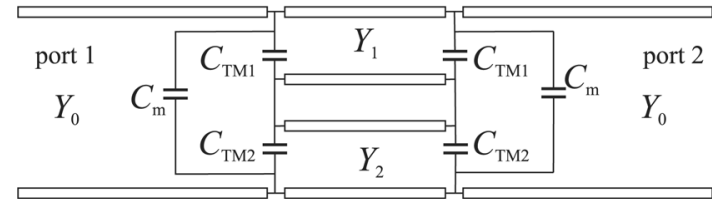

(b)

Fig. 3. (a) Unit cell of a perfect metal screen with groups of three symmetrically located slits per period (example of compound grating). (b) Equivalent circuit model for this structure and normal incidence.

It is worth investigating if our capacitively loaded transmission line model also accounts for the above peculiar phenomenology. For this purpose, the example case shown in Fig. 3(a) will be considered. In this case, the compound transmission grating has a unit cell containing three closely spaced slits. The slits can be empty (as in [32], [36] and [38]-[40]), but they can also be filled with some isotropic linear dielectric materials to increase control on the phenomenon (the case treated in [37]). Nevertheless, the geometry and distribution of the dielectrics must be chosen so that the plane $B B^{\prime}$ in Fig. 3(a) is a symmetry plane. For normal incidence, assuming that the impinging electric field is polarized along the direction normal to the slits, this plane is a virtual electric wall. This problem was considered previously by the authors in [29] assuming empty slits. However, the model in [29] needs some improvements to account accurately for the details of the observed transmission spectra.

Following the same rationale as in Section II, the equivalent circuit proposed for the structure is shown in Fig. 3(b). The two potentially resonant transmission line sections loaded with a capacitive network (involving the capacitances $C_{1}, C_{2}$, and $C_{\mathrm{m}}$ ) have characteristic admittances given by $Y_{1}=\sqrt{\varepsilon_{r 1}} W /\left(a_{1} \eta_{0}\right)$ and $Y_{2}=2 \sqrt{\varepsilon_{r 2}} W /\left(a_{2} \eta_{0}\right)$, while the characteristic admittance of the input and output lines is $Y_{0}=2 W /\left(d \eta_{0}\right)$. It should be noted that this model is different from that proposed in [29] due to the addition of the mutual coupling capacitance $C_{\mathrm{m}}$ and the presence of a dielectric filling the slits. The consequences of including the coupling capacitance will be discussed later. The equivalent circuit includes two transmission lines (and not three) to model the three slits system because of the electric wall induced by the symmetry with respect to the $B B^{\prime}$ plane. It is worth mentioning that, for normal incidence, the same equivalent circuit would be valid for the case of four slits per period since 


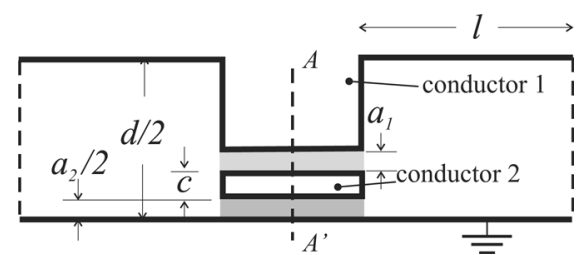

Fig. 4. 2-D electrostatic problem used to determine the capacitances of the model in Fig. 3.

the symmetry plane makes it possible to consider only two different slits per unit cell for both cases. From a qualitative point of view, this means that the same effects should arise in systems with three slits $(J=3$ following the notation in [32]) and with four slits $(J=4)$. Thus, the same number of dips should be obtained for the cases $J=3$ and $J=4$. Similarly, the case $J=2$ (two identical slits per period) should not offer special novelties with respect to the simple grating studied in Section II; namely, no transmission dips are expected for the case $J=2$. The extension of the circuit model in Fig. 3(b) to higher values of $J$ is rather obvious: $N$ transmission lines should be used for $J=2 N-1$ or $J=2 N$, and these lines should be loaded with a capacitive network similar to the one depicted in Fig. 3(b), but considering $N$ edge capacitances (one for each of the involved transmission lines) plus $N$ mutual capacitances accounting for all the possible capacitive coupling. However, for the sake of simplicity, we will concentrate on the case $J=3$ (or $J=4$ ) since the essential points of the model are clearly stated using this case.

The physical meaning of the capacitances shown in Fig. 3(b) is somewhat evident: two edge capacitances, $C_{1}$ and $C_{2}$, to account for the stray electric fields near and (mostly) outside the slits plus a nonnegligible coupling capacitance $C_{\mathrm{m}}$ to account for the close proximity between the slits. If the working frequency is close to the onset frequency of the first TM mode in the input and output waveguides, the edge capacitances would behave as in (6), having a singular behavior at the Rayleigh-Wood anomaly frequency. However, when Fabry-Pérot resonances are the relevant phenomenon, these capacitances will be almost frequency independent for frequencies below and far from the above onset frequency (although an extraordinary transmission peak near the onset of the first TM mode is also expected for the compound grating structure). The values of the capacitances in our model can be extracted from the solution of the 2-D electrostatic problem posed in Fig. 4. The region where Laplace's equation has to be solved is bounded by two magnetic walls, ground conductor, and surface of conductors 1 and 2 . In fact, thanks to the symmetry of the structure with respect to the magnetic-wall plane $A A^{\prime}$ in Fig. 4, only the right half of that structure has to be considered. The three capacitances will then be obtained solving the electrostatic problem posed in Fig. 4 under two independent voltage excitations. In particular, one of the excitations imposes $1 \mathrm{~V}$ for conductor 1 , while conductor 2 is grounded and the other excitation imposes $1 \mathrm{~V}$ for both conductors. From the total charge stored on each conductor in those two cases and from the values of the three ideal parallel-plate capacitors (namely, without edge capacitance) that can be identified in Fig. 4 (region between conductor 1 and 2, region between conductor 2 and ground, and region between conductor 1 and ground outside the slits), it is a simple exercise to extract the values of $C_{1}, C_{2}$, and $C_{\mathrm{m}}$. It should be noticed that $C_{\mathrm{m}}$ might be negative.

Once the values of the capacitances are known, the scattering parameters of our equivalent circuit are trivially computed. It could be considered that the two transmission line sections loaded with shunt capacitances are series connected in such a way that the impedance matrix is just the summation of the impedance matrices of each section plus the loading shunt connection of a resistance (the characteristic impedance of the output transmission line $Y_{0}^{-1}$ ) and the coupling capacitance $C_{\mathrm{m}}$. However, in order to exploit symmetries, it is convenient to again use even/odd excitations. The symmetry of such excitations together with the symmetry of the structure leads to the appearance of electric (odd excitation) or magnetic (even excitation) walls at the vertical symmetry plane $A A^{\prime}$ in Fig. 3(b).

1) Odd Excitation Case: For this excitation, the $A A^{\prime}$ plane is an electric wall. Thus, short-circuited transmission line sections of length $h / 2$ are shunt connected to the capacitances $C_{1}$ and $C_{2}$. The resulting impedances are series connected and the whole is shunt connected to the coupling capacitance $C_{\mathrm{m}}$. The odd excitation admittance $Y_{L}^{o}$ loading the input transmission line (characteristic admittance $Y_{0}$ ) is then given by

$$
\begin{aligned}
Y_{L}^{o}=\left[\frac{1}{\mathrm{j} \omega C_{\mathrm{TM}_{1}}-\mathrm{j} Y_{1} \cot \left(\theta_{1} / 2\right)}\right. \\
\left.\quad+\frac{1}{\mathrm{j} \omega C_{\mathrm{TM}_{2}}-\mathrm{j} Y_{2} \cot \left(\theta_{2} / 2\right)}\right]^{-1}+\mathrm{j} \omega C_{\mathrm{m}} .
\end{aligned}
$$

2) Even Excitation Case: In this case, the middle vertical symmetry plane $A A^{\prime}$ is a magnetic wall. Thus, open-circuited transmission line sections are shunt connected to the capacitances $C_{1}$ and $C_{2}$. The admittance loading the input transmission line is now

$$
\begin{aligned}
Y_{L}^{e}=\left[\frac{1}{\mathrm{j} \omega C_{\mathrm{TM}_{1}}+\mathrm{j} Y_{1} \tan \left(\theta_{1} / 2\right)}\right. \\
\left.\quad+\frac{1}{\mathrm{j} \omega C_{\mathrm{TM}_{2}}+\mathrm{j} Y_{2} \tan \left(\theta_{2} / 2\right)}\right]^{-1}+\mathrm{j} \omega C_{\mathrm{m}} .
\end{aligned}
$$

From transmission line theory, even/odd reflection coefficients can be defined as

$$
S_{11}^{e / o}=\frac{Y_{0}-Y_{L}^{e / o}}{Y_{0}+Y_{L}^{e / o}}
$$

which can be introduced in (3) to obtain the desired scattering parameters $S_{11}$ (reflection) and $S_{12}$ (transmission).

The expressions (3) and (8)-(10) can now be used to reproduce the numerical and/or experimental results reported in previous publications. However, it should be highlighted that the novelty of the present approach does not lie in the development of a different numerical method to generate those results. Instead, our goal is the introduction of a new methodology capable of raising qualitative and semiquantitative predictions that are not obvious at all from the numerical approaches. In this new frame, it is then possible to know a priori how each geometrical parameter affects the transmission/reflection response 
of the grating. In order to illustrate the above fact, the transmission spectra reported in previous papers on the subject will be analyzed at the light of our equivalent-circuit model. It is obvious from (3) that total transmission can be attained provided $S_{11}^{e}=-S_{11}^{o}$ since this condition implies $S_{11}=0$, and from power conservation, $\left|S_{12}\right|=1$. Conversely, all the impinging power is reflected, thus giving place to transmission dips if $S_{11}^{e}=S_{11}^{o}$. From (10), these conditions can be turned into the following requirements for the normalized values of the even/odd loading admittances $\bar{Y}_{L}^{e / o}=Y_{L}^{e / o} / Y_{0}$.

1) Total transmission condition

$$
\bar{Y}_{L}^{o}=1 / \bar{Y}_{L}^{e}
$$

2) Total reflection (transmission dip) condition

$$
\bar{Y}_{L}^{o}=\bar{Y}_{L}^{e}
$$

A detailed examination of $Y_{L}^{o / e}$ given in (8) and (9) reveals that these functions are monotonic functions whose zeros and poles alternate (Foster's theorem), and this is essential to making sure that conditions (11) and (12) can be satisfied for an infinite number of discrete frequency points. Nothing more specific can be said about the distribution of those frequency points for arbitrary values of the relative permittivity of the dielectrics inside the slits. However, some important qualitative predictions can be made for empty slits. In particular, our attention will focus on the three slits case $(J=3)$ studied in [32]. The slits of this structure are empty in such a way that $\theta_{1}=\theta_{2} \equiv \theta$. The transmission spectrum reported in [32] is similar, in some aspects, to the spectrum corresponding to the single slit case. It has total transmission peaks at those frequencies for which Fabry-Pérot resonances are expected, but the simple transmission peaks observed in the single slit case are now substituted by two closely spaced total transmission peaks separated by a transmission zero.

Next it will be shown how our model can anticipate this numerically predicted result. The inspection of the total reflection condition (12) tells us that this condition is unaffected by the value of $C_{\mathrm{m}}$. However, it is strongly dependent on the values of the ratios $\omega C_{\mathrm{TM}_{1}} / Y_{1}$ and $\omega C_{\mathrm{TM}_{2}} / Y_{2}$. As a first approximation, we assume that the edge capacitances are proportional to the line admittances in such a way that the above ratios are identical. This is equivalent to say that the excess lengths associated with the two edge capacitances are identical. In this situation, simple algebraic manipulations leads to the following condition for total reflection:

$$
\tan (\theta / 2)+\cot (\theta / 2)=0 .
$$

It is important to note that this condition would also be obtained for any number of transmission lines in the model of Fig. 3(b) provided that the excess lengths for all the transmission lines are identical. Obviously, (13) has no real solutions, which means that no transmission dips would appear for any number of slits per period. Since numerical simulations and experiments do show transmission dips, there should be something wrong in our first assumption. A deeper look at the problem indicates that the previous approximation cannot be true as long as the environment of each of the two slits in Fig. 3(a) is not identical. The use of a 2-D electrostatic solver to determine the values of $C_{\mathrm{TM}_{1}}$ and $C_{\mathrm{TM}_{2}}$ for the examples treated in [32] confirms that there is a significant difference between these capacitances. The situation can be better understood if the total reflection condition is written in the following explicit manner using (8), (9), and (12):

$$
\begin{aligned}
& {\left[\frac{1}{\omega C_{\mathrm{TM}_{1}}-Y_{1} \cot (\theta / 2)}+\frac{1}{\omega C_{\mathrm{TM}_{2}}-Y_{2} \cot (\theta / 2)}\right]} \\
& \quad=\left[\frac{1}{\omega C_{\mathrm{TM}_{1}}+Y_{1} \tan (\theta / 2)}+\frac{1}{\omega C_{\mathrm{TM}_{2}}+Y_{2} \tan (\theta / 2)}\right]
\end{aligned}
$$

where empty slits have been considered $\left(\theta_{1}=\theta_{2}=\theta\right)$. Note that all the terms in (14) are real. In the frequency ranges where the electrical lengths of the lines take values around $\theta=(2 n+$ 1) $\pi(n=0,1, \ldots)$, we can use the concept of equivalent electrical length of the transmission lines, denoted here as $\tilde{\theta}$, for the left-hand side (LHS) of (14). This equivalent electrical length is obtained taking into account that, for the frequency range of interest (around the zeros of the cotangent functions), it is possible to approximate the characteristic functions in the following way:

$$
-Y_{1,2} \cot \left(\tilde{\theta}_{1,2} / 2\right)=\omega C_{\mathrm{TM}_{1,2}}-Y_{1,2} \cot (\theta / 2)
$$

provided $\tilde{\theta}_{1,2} \approx \theta+\Delta \theta_{1,2}$. The equivalent length is then the sum of the physical electrical length $\theta=\left(\omega / c_{0}\right) h$ plus the excess electrical length $\left.\Delta \theta_{1,2}=\left(2 \omega C_{\mathrm{TM}_{1,2}}\right) / Y_{1,2}\right)$. Writing the LHS bracket in (14) now as

$$
\frac{\tan \left(\tilde{\theta}_{1} / 2\right)}{Y_{1}}+\frac{\tan \left(\tilde{\theta}_{2} / 2\right)}{Y_{2}}
$$

it can easily be seen that this expression has two relatively close poles corresponding to those frequencies $f_{1}$ and $f_{2}$ for which the equivalent electrical length of one of the two lines is exactly $\pi$ (or an odd number of times $\pi$ ). Clearly, in the frequency range between $f_{1}$ and $f_{2}$, this LHS member ranges from $-\infty$ to $\infty$. In contrast with that behavior, in this same frequency range, the RHS member of (14) is a smooth function that takes relatively small values (due to the fact that the tangent functions appearing in this member will take large values in that frequency range). The combination of the above two behaviors will ensure that condition (14) is satisfied for a single frequency value between $f_{1}$ and $f_{2}$, and more precisely, very close to $\left(f_{1}+f_{2}\right) / 2$. A similar situation occurs for electrical lengths around $2 n \pi$, but in this case, it will be the right-hand side (RHS) member in (14) of the function providing the two poles, whereas the LHS member will behave smoothly. In brief, since the equivalent electrical lengths of the two transmission lines are different, one transmission zero is ensured in the frequency region corresponding to each of the Fabry-Pérot orders. This argument can be extended to $N$ transmission lines in the model in Fig. 3(b) in such a way that, if all the electrical lengths are different, $(N-1)$ total reflection points should appear.

To study the total transmission condition (11), for simplicity it will first be assumed that $C_{\mathrm{m}}=0$ (as was implicitly done in [29]). In this case, the LHS member of (11) has the poles in 
the region where the RHS member takes small values in such a way that two solutions to (11) should be found near the frequencies $f_{1}$ and $f_{2}$. Thus, the model without $C_{\mathrm{m}}$ predicts two total transmission peaks located around the transmission zero at approximately the same frequency distance below and above (see [29] for an example). However, numerical simulations and experiments yield total reflection frequency located somewhere between $f_{1}$ and $f_{2}$, but relatively far from $\left(f_{1}+f_{2}\right) / 2$ [32], [37]-[40]. The off-centered position of the transmission zero with respect to the theoretical Fabry-Pérot transmission band comes in our approach from the presence of $C_{\mathrm{m}}$. The displacement of the transmission peaks when $C_{\mathrm{m}}$ is considered (remember that $C_{\mathrm{m}}$ does not affect the position of the total reflection points) can be toward higher or lower frequencies depending on the sign of this parameter. It can then be concluded that, although the existence of the transmission zeros can be explained on the basis of the differences of equivalent electrical lengths of the transmission lines involved in the model, the exact position of the transmission zeros with respect to the transmission maxima is related to the particular value of the mutual coupling capacitance.

\section{VALIDATION RESULTS FOR LOSSLESS AND LOSSY COMPOUND GRATINGS}

In this section, the equivalent-circuit model previously proposed for compound gratings will be validated by comparing the transmission spectra provided by this circuit model versus the spectra obtained via mode-matching full-wave computations (for lossless structures), as well as measured transmission spectra. In this latter case, losses must be included in our model for an accurate comparison of both critical frequencies and transmission losses. Assuming that losses can be accounted for by the strong skin-effect approach (which is actually the case for the example used in this section), the introduction of losses in our model is relatively simple. First, the transmission lines involved in the model having characteristic admittances $Y_{1}$ and $Y_{2}$ must be considered lossy, which implies that now $Y_{1}$ and $Y_{2}$ are complex quantities to account for the addition of a resistance pul in the transmission line model. Since the involved transmission lines are simple parallel-plate waveguides, the pul resistance in each line is given by

$$
R_{\mathrm{pul}, 1}=\frac{1}{W} \sqrt{\frac{2 \omega \mu_{0}}{\sigma}} \quad R_{\mathrm{pul}, 2}=R_{\mathrm{pul}, 1} / 2
$$

with $\sigma$ being the conductivity of the metal. For the dimensions considered in our examples, the effect of the internal inductance can be neglected. Obviously the propagation constants of the parallel-plate transmission lines that model the slits also become complex, as well as the electrical lengths of these transmission lines. The inclusion of (15) accounts for losses inside the slits, but additional losses will come from the current flowing on the vertical surfaces of the grating [see Fig. 3(a)]. Since the surface current density on this surface is uniform, its skin effect resistance is readily found to be

$$
R=\sqrt{\frac{\omega \mu_{0}}{2 \sigma}} \frac{\left(d-a_{2}-2 a_{1}\right)}{2 W} .
$$

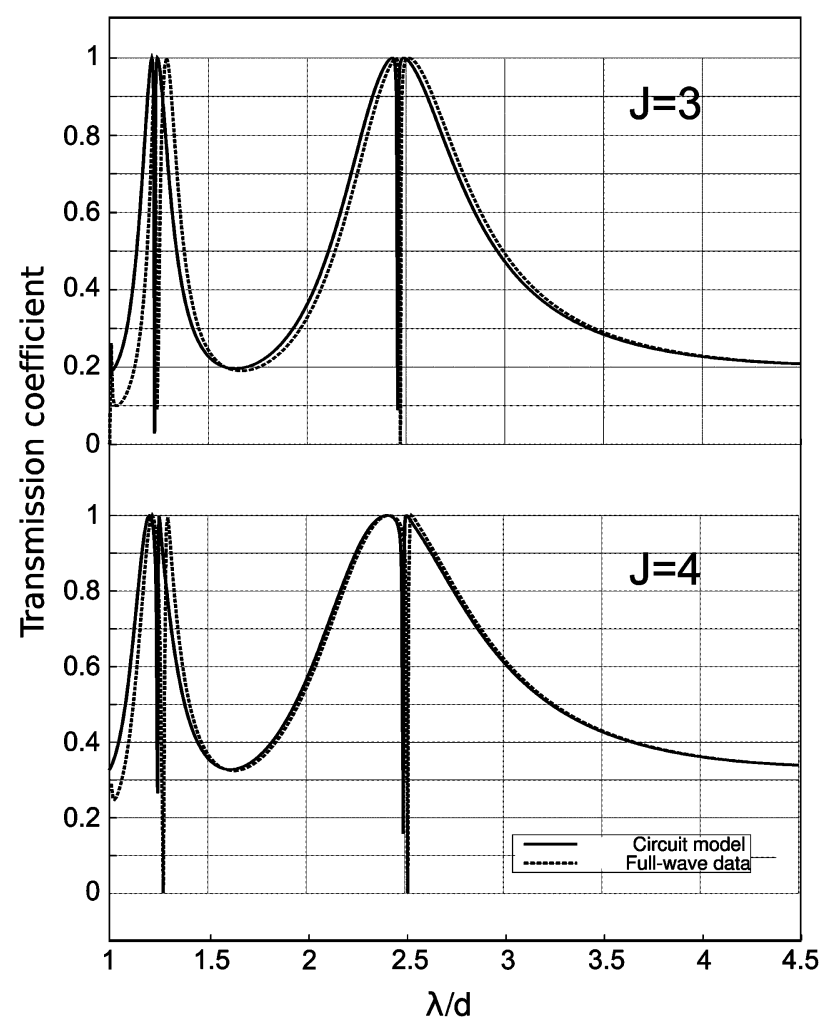

Fig. 5. Transmission spectra for two lossless compound gratings. Dimensions are the same as in [32, Fig. 2]: $d=10 \mathrm{~mm}, h=11.4 \mathrm{~mm}, a=0.8 \mathrm{~mm}$, $c=0.8 \mathrm{~mm}$. Solid lines: circuit model predictions; dashed lines: numerical data. The circuit parameters used to generate these curves are: (top) $Y_{0}=$ $0.531 \Omega^{-1}, Y_{1}=3.318 \Omega^{-1}, Y_{2}=6.636 \Omega^{-1}, C_{1}=3.79 \mathrm{pF}, C_{2}=$ $10.95 \mathrm{pF}$, and $C_{\mathrm{m}}=0.274 \mathrm{pF}$ and (bottom) $Y_{0}=0.531 \Omega^{-1}, Y_{1}=Y_{2}=$ $3.318 \Omega^{-1}, C_{1}=4.89 \mathrm{pF}, C_{2}=6.88 \mathrm{pF}$, and $C_{\mathrm{m}}=-1.307 \mathrm{pF}$.

This resistance is series connected with the input and output transmission lines (characteristic admittance $Y_{0}$ ). After this element is included in the even and odd admittances in (8) and (9), which represent only a few additional lines in our computer code, our approach is ready to deal with lossy conductors under the skin-effect regime (this approximation should be adequately adapted to account for optical frequencies since, in that regime, metals are described by frequency-dependent complex permittivities rather than by real conductivities).

A first numerical validation will be carried out for a lossless perfect-conductor compound grating having $J=3$ and $J=4$ with the dimensions used in [32]. Since in that paper the conductors were considered metals characterized by a complex refractive index, the numerical mode-matching code has been run again to obtain the transmission spectrum in the perfect conductor limit. For the structures under study, the capacitances to be employed in the equivalent-circuit model have been obtained using a 2-D electrostatic solver. The computed data from the numerical method are plotted in Fig. 5 along with those provided by the equivalent-circuit model. A good agreement is found for the Fabry-Pérot regime, including fine details about the relative position of the dips in the transmission bands. Note that the transmission dips are off centered. Displacement to lower wavelengths ( $J=3$, first Fabry-Pérot resonance) are due to a positive value of $C_{\mathrm{m}}$, whereas displacement to higher wavelengths $(J=4)$ is related to a negative value of $C_{\mathrm{m}}$. The narrow 


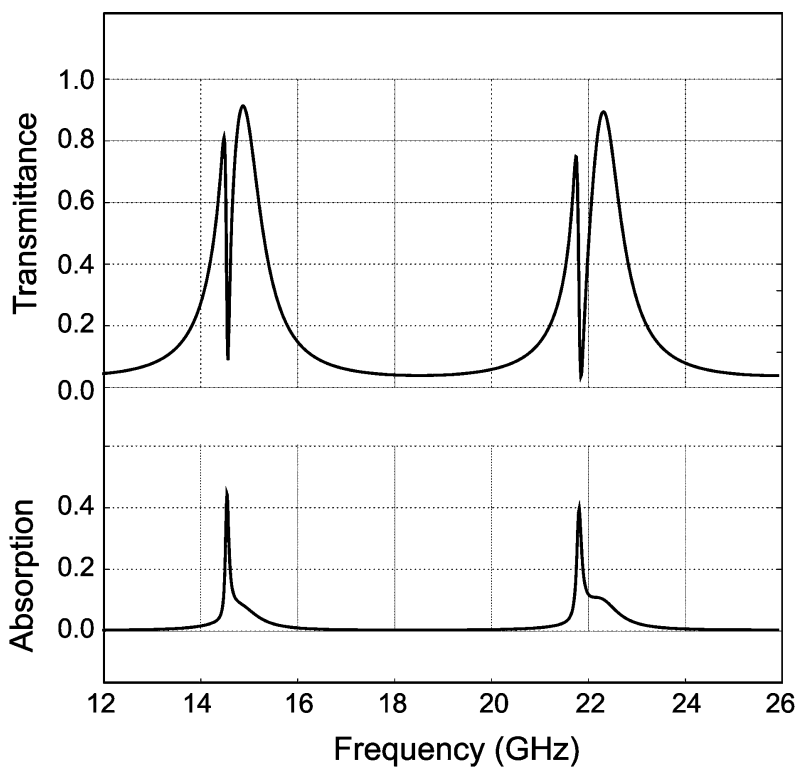

Fig. 6. Transmission spectrum for the structure considered in [38, Fig. 2] obtained using the circuit model in this paper. Perfect agreement with the numerical simulations and measured data in [38] has been found.

transmission peak very close to $\lambda / d=1$ is not captured by our circuit model simulation because we did not include the singular behavior of the capacitances in this simulation (this is the extraordinary transmission peak), although it can easily be carried out as already shown in Section II-B for the single slit case. For this same reason, the agreement between circuit theory model and full-wave mode matching results deteriorates near $\lambda / d \gtrsim 1$. If the Fabry-Pérot band corresponding to $\lambda \simeq h$ were not so close to the onset frequency of the first grating lobe (cutoff frequency of the first TM higher order mode), which would occur if $h$ were not so close to $d$ as in the example, the agreement would have been much better for this Fabry-Pérot order. More simulations for the lossless case have been carried out to check our model with very good results in all the cases.

In Fig. 6, the experimental data reported in [38, Fig. 2] have been reproduced with our equivalent-circuit model. The lossy nature of the real conductors was introduced in our model via the conductivity value of aluminum in (15) and (16). The comparison of our data with the experimental results reported in [38] gives an excellent agreement even for the absorption level. The agreement is even better than in the previous example because now the thickness of the screen ( $h$ in this paper, but $t$ in [38]) is not so close to the period of the structure (in this case, $h \approx 2 d$ ). This comparison also supports the validity of our model for the case of actual lossy metals, at least at microwave frequencies, when the skin regime is dominant.

As a final example, it will be considered the compound gratings with dielectric-filled slits previously studied in [37]. The results reported in [37, Fig. 2] have been reproduced with our equivalent-circuit model in Fig. 7. In particular, the results in this plot correspond to the case having the central slit (out of the three identical slits per unit cell) filled with a dielectric with different relative permittivity. It can be verified that the agreement with the data in [37, Fig. 2] is excellent. The use of dielectric inside the slits was proposed in [37] as a method to manipulate



Fig. 7. Transmission spectrum for the structures considered in [37, Fig. 2] having a dielectric in the central slit. The electrical parameters are the same used in Fig. 5 $(J=3)$, except the characteristic admittance $Y_{2}$, which is $\sqrt{\varepsilon_{r}}$ times $Y_{2}$ in vacuum. Very good agreement with the numerical simulations in [37] is obtained.

the transmission pattern of the grating. Our equivalent-circuit approach provides a good tool to tailor (of course with limitations) the transmission spectrum since it can predict how each parameter will affect the distribution of maxima and minima.

\section{CONCLUSION}

The transmission of electromagnetic waves through simple and compound 1-D diffraction gratings has been analyzed at the light of conventional transmission line theory. The concepts reported in this paper provide a relatively simple explanation of an apparently complex physical phenomenon. The simplicity of the proposed theory would qualify the approach as a sound pedagogical contribution to the understanding of the phenomenon, but the most important benefit of the reported theory from an engineering point of view is that it provides a methodology to analyze structures and/or to conceive new devices based on the studied physical phenomenon. The design tasks can be considerably simplified since the effect of each of the elements of the equivalent-circuit model on the transmission response is qualitatively known a priori. From a scientific point of view, the model gives an alternative explanation based on conventional impedance matching concepts rather than on surface plasmon Bloch-like waves. Moreover, the treatment in this paper together with the model reported in [24] set up a unified treatment of the transmission through 2-D and 1-D arrays of holes or slits. 


\section{REFERENCES}

[1] T. W. Ebbesen, H. J. Lezec, H. F. Ghaemi, T. Thio, and P. A. Wolff, "Extraordinary optical transmission through sub-wavelength hole arrays," Nature, vol. 391, pp. 667-669, Feb. 1998.

[2] F. J. García-de-Abajo, "Colloquium: Light scattering by particle and hole arrays," Rev. Mod. Phys., vol. 79, pp. 1267-1290, Oct.-Dec. 2007.

[3] C. Genet and T. W. Ebbesen, "Light in tiny holes," Nature, vol. 445, pp. 39-46, Jan. 2007.

[4] A. Nahata, T. Matsui, A. Agrawal, and Z. V. Vardeny, "Transmission resonances through aperiodic arrays of subwavelength apertures," $\mathrm{Na}$ ture, vol. 446, pp. 517-521, 2007.

[5] H. J. Lezec, A. Degiron, E. Devaux, R. A. Linke, L. Martín-Moreno, F. J. García-Vidal, and T. W. Ebbesen, "Beaming light from a subwavelength aperture," Science, vol. 297, pp. 820-822, 2002.

[6] A. A. Oliner and D. R. Jackson, "Leaky surface-plasmon theory for dramatically enhanced transmission through a subwavelength aperture-Part I: Basic features," in IEEE AP-S Int. Symp., Jun. 22-27, 2003, vol. 2, pp. 1091-1094.

[7] D. R. Jackson, T. Zhao, J. T. Williams, and A. A. Oliner, "Leaky surface-plasmon theory for dramatically enhanced transmission through a sub-wavelength aperture-Part II: Leaky-wave antenna model," in IEEE AP-S Int. Symp., Jun. 22-27, 2003, vol. 2, pp. 1095-1098.

[8] S. S. Akarca-Biyikli, I. Bulu, and E. Ozbay, "Enhanced transmission of microwave radiation in one-dimensional metallic gratings with subwavelength aperture," Appl. Phys. Lett., vol. 85, no. 7, pp. 1098-2000, Aug. 2004.

[9] F. J. García-de-Abajo, "Light transmission through a single cylindrical hole in a metallic film," Opt. Exp., vol. 10, no. 25, pp. 1475-1484, 2002.

[10] J. M. Brok and H. P. Urbach, "Extraordinary transmission through 1, 2 and 3 holes in a perfect conductor, modelled by a mode expansion technique," Opt. Exp., vol. 14, no. 7, pp. 2552-2572, Apr. 2006.

[11] G. F. Koch and K. S. Kolbig, "The transmission coefficient of elliptical and rectangular apertures for electromagnetic waves," IEEE Trans. Antennas Propag., vol. AP-16, no. 1, pp. 78-83, Jan. 1968.

[12] J. M. Jin and J. M. Volakis, "Electromagnetic scattering by and transmission through a three-dimensional slot in a thick conducting plane," IEEE Trans. Antennas Propag., vol. 39, no. 4, pp. 543-550, Apr. 1991.

[13] J. B. Pendry, L. Martín-Moreno, and F. J. Garcia-Vidal, "Mimicking surface plasmons with structured surfaces," Science, vol. 305, pp. 847-848, Aug. 2004.

[14] F. J. García-de-Abajo and J. J. Sáenz, "Electromagnetic surface modes in structured perfect-conductor surfaces," Phys. Rev. Lett., vol. 95, pp. 233901-1-233901-4, Dec. 2005.

[15] C. R. Williams, S. R. Andrews, S. A. Maier, A. I. FernándezDomínguez, L. Martín-Moreno, and F. J. García-Vidal, "Highly confined guiding of terahertz surface plasmon polaritons on structured metal surfaces," Nature Photon., vol. 2, pp. 175-179, Mar. 2008.

[16] M. J. Lockyear, A. P. Hibbins, and J. R. Sambles, "Microwave surfaceplasmon-like modes on thin metamaterials," Phys. Rev. Lett., vol. 102, p. 073901, Feb. 2009.

[17] M. Beruete, M. Sorolla, I. Campillo, J. S. Dolado, L. Martín-Moreno, J. Bravo-Abad, and F. J. García-Vidal, "Enhanced millimeter wave transmission through quasioptical subwavelength perforated plates," IEEE Trans. Antennas Propag., vol. 53, no. 6, pp. 1897-1903, Jun. 2005.

[18] R. E. Collin, Field Theory of Guided Waves. New York: IEEE Press, 1971.

[19] W. L. Barnes, A. Dereux, and T. W. Ebbesen, "Surface plasmon subwavelength optics," Nature, vol. 424, pp. 824-830, Aug. 2003.

[20] M. M. J. Treacy, "Dynamical diffraction explanation of the anomalous transmission of light through metallic gratings," Phys. Rev. B, Condens. Matter, vol. 66, pp. 195105-1-195105-11, 2002.

[21] H. J. Lezec and T. Thio, "Diffracted evanescent wave model for enhanced and suppressed optical transmission through subwavelength hole arrays," Opt. Exp., vol. 12, no. 16, pp. 3629-3651, Aug. 2004.

[22] F. J. García de Abajo, R. Gómez-Medina, and J. J. Sáenz, "Full transmission through perfect-conductor subwavelength hole arrays," Phys. Rev. E, vol. 72, pp. 016608-1-016608-4, 2005.

[23] F. Medina, F. Mesa, and R. Marqués, "Equivalent circuit model to explain extraordinary transmission," in IEEE MTT-S Int. Microw. Symp., Atlanta, GA, Jun. 15-20, 2008, pp. 213-216.

[24] F. Medina, F. Mesa, and R. Marqués, "Extraordinary transmission through arrays of electrically small holes from a circuit theory perspective," IEEE Trans. Microw. Theory Tech., vol. 56, no. 12, pp. 3108-3120, Dec. 2008.
[25] R. Gordon, "Bethe's aperture theory for arrays," Phys. Rev. A, vol. 76, 2007, Art. ID 053806.

[26] Y. Pang, A. N. Hone, P. P. M. So, and R. Gordon, "Total optical transmission through a small hole in a metal waveguide screen Bethe's aperture," Opt. Exp., vol. 17, no. 6, pp. 4433-4441, 2009.

[27] A. A. Kirilenko and A. O. Perov, "On the common nature of the enhanced and resonance transmission through the periodical set of holes," IEEE Trans. Antennas Propag., vol. 56, no. 10, pp. 3210-3216, Oct. 2008.

[28] N. G. Don, A. A. Kirilenko, and S. L. Senkevich, "New type of eigenoscillations and total-transmission resonance through an iris with below-cutoff hole in a rectangular waveguide," Radiophys. Quantum Electron., vol. 51, no. 2, pp. 101-108, Feb. 2008.

[29] F. Medina, D. C. Skigin, and F. Mesa, "Extraordinary transmission through slits from a microwave engineering perspective," in Proc. 38th Eur. Microw. Con., Amsterdam, The Netherlands, Oct. 2008, pp. 702-705.

[30] J. A. Porto, F. J. García-Vidal, and J. B. Pendry, "Transmission resonances on metallic gratings with very narrow slits," Phys. Rev. Lett., vol. 83, no. 14, pp. 2845-2848, Oct. 1999.

[31] F. J. García-Vidal and L. Martín-Moreno, "Transmission and focusing of light in one-dimensional periodically nanostructured metals," Phys. Rev. B, Condens. Matter, vol. 66, pp. 155412-1-155412-10, 2002.

[32] D. C. Skigin and R. Depine, "Transmission resonances of metallic compound gratings with subwavelength slits," Phy. Rev. Lett., vol. 95, pp. 217402-1-217402-4, 2005.

[33] Y. Takakura, "Optical resonance in a narrow slit in a thick metallic screen," Phys. Rev. Lett., vol. 86, no. 24, pp. 5601-5603, Jun. 2001.

[34] F. Yang and J. R. Sambles, "Resonant transmission of microwaves through a narrow metallic slit," Phys. Rev. Lett., vol. 89, no. 6, pp. 063901-1-063901-4, Aug. 2002.

[35] J. R. Suckling, A. P. Hibbins, M. J. Lockyear, T. W. Preist, J. R. Sambles, and C. R. Lawrence, "Finite conductance governs the resonance transmission of thin metal slits at microwave frequencies," Phys. Rev. Lett., vol. 92, no. 14, pp. 147401-1-147401-4, Apr. 2004.

[36] D. C. Skigin and R. Depine, "Narrow gaps for transmission through metallic structured gratings with subwavelength slits," Phys. Rev. E, vol. 74, pp. 046606-1-046606-6, 2006.

[37] D. C. Skigin, H. Loui, Z. Popovic, and E. F. Kuester, "Bandwidth control of forbidden transmission gaps in compound structures with subwavelength slits," Phys. Rev. E, vol. 76, pp. 016604-1-016604-6, 2007.

[38] A. P. Hibbins, I. R. Hooper, M. J. Lockyear, and J. R. Sambles, "Microwave transmission of a compound metal grating," Phys. Rev. Lett., vol. 96, pp. 257402-1-257402-4, 2006.

[39] Y. G. Ma, X. S. Rao, G. F. Zhang, and C. K. Ong, "Microwave transmission modes in compound metallic gratings," Phys. Rev. B, vol. 76, pp. 085413-1-085413-5, Aug. 2007.

[40] M. Navarro-Cía, D. C. Skigin, M. Beruete, and M. Sorolla, "Experimental demonstration of phase resonances in metallic compound gratings with subwavelength slits in the millimeter wave regime," App. Phys. Lett., vol. 94, pp. 091107-1-091107-3, Mar. 2009.

[41] A. A. Kirilenko, private communication.

[42] V. G. Sologub, V. P. Schestopalov, and G. G. Polovnikov, "Diffraction of electromagnetic waves on the grating with narrow slits," (in Russian) J. Tech. Phys., vol. 37, no. 4, pp. 667-679, 1967.

[43] V. G. Sologub and V. P. Schestopalov, "The resonance phenomena at the diffraction of the $H$-polarized waves on the gratings of metal bars," (in Russian) J. Tech. Phys., vol. 38, no. 9, pp. 1505-1520, 1968.

[44] S. A. Masalov, Y. K. Sirenko, and V. P. Schestopalov, "The solution of the problem of the plane wave diffraction on the knife-type grating with compound structure of a period," (in Russian) Radiotek. Electron., vol. XXIII, no. 3, pp. 481-487, 1978.

[45] M. Guglielmi and A. A. Oliner, "Multimode network description of a planar periodic metal-strip grating at a dielectric interface-Part I: Rigorous network formulations," IEEE Trans. Microw. Theory Tech., vol. 37, no. 3, pp. 535-541, Mar. 1989.

[46] M. Guglielmi and A. A. Oliner, "Multimode network description of a planar periodic metal-strip grating at a dielectric interface-Part II: Small-aperture and small-obstacle solutions," IEEE Trans. Microw. Theory Tech., vol. 37, no. 3, pp. 542-552, Mar. 1989.

[47] A. Fernández-Prieto, F. Medina, and F. Mesa, "Microstrip circuit analog of a complex diffraction phenomenon," Appl. Phys. Lett., vol. 95, pp. 021108-1-021108-3, Jul. 2009. 


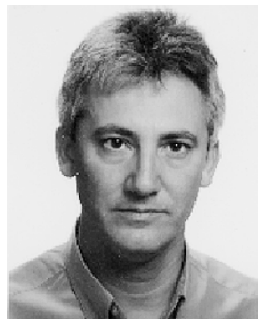

Francisco Medina (M'90-SM'01-F'10) was born in Puerto Real, Cádiz, Spain, in November 1960. He received the Licenciado and Doctor degrees from the University of Seville, Seville, Spain, in 1983 and 1987 respectively, both in physics.

From 1986 to 1987, he spent the academic year with the Laboratoire de Microondes de l'ENSEEIHT, Toulouse, France. From 1985 to 1989, he was an Assistant Professor with the Department of Electronics and Electromagnetism, University of Seville, where, in 1990, he became an Associate Professor of electromagnetism. He is currently a Full Professor of electromagnetism (since July 2009) and Head of the Microwaves Group. He is on the Editorial Board of the International Journal of RF and Microwave Computer-Aided Engineering. $\mathrm{He}$ is a reviewer for approximately 24 Institution of Electrical Engineers (IEE), U.K., and American Physics Society journals. His research interest includes analytical and numerical methods for guiding, resonant, and radiating structures, passive planar circuits, periodic structures, and the influence of anisotropic materials (including microwave ferrites) on such systems. He is also interested in artificial media modeling and design and extraordinary transmission phenomena.

Dr. Medina is a reviewer for the IEEE TRANSACTIONS ON MicrowaVE THEORY AND TECHNIQUES, as well as other IEEE publications. He has been a member of the Technical Program Committees (TPCs) of several international and local conferences and has organized a few conferences and workshops. He is a Fellow of the Massachusetts Institute of Technology (MIT) Electromagnetics Academy. He was the recipient of a Spanish Ministerio de Educación y Ciencia (MEC) Research Scholarship and a French Ministére de la Recherche et la Technologie Scholarship.

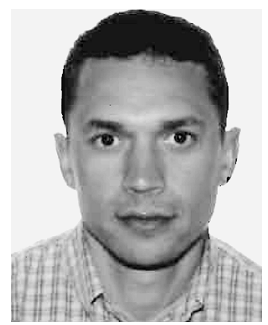

Francisco Mesa (M’93) was born in Cádiz, Spain, in April 1965. He received the Licenciado and Doctor degrees in physics from the Universidad de Sevilla, Seville, Spain, in 1989 and 1991, respectively.

$\mathrm{He}$ is currently an Associate Professor with the Departamento de Física Aplicada 1, Universidad de Sevilla. His research interests focus on electromagnetic propagation/radiation in planar structures. He is also involved with extraordinary transmission and metamaterials.

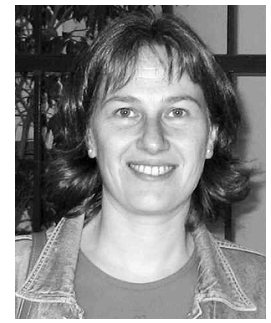

Diana C. Skigin (M'06) received the Licenciada en Ciencias Físicas (M.S.) degree in physics and Ph.D. degree in physics from the Faculty of Exact and $\mathrm{Na}$ ture Sciences, University of Buenos Aires, Buenos Aires, Argentina, in 1992 and 1997, respectively.

In 1992, she began teaching with the University of Buenos Aires, where she is currently a Professor. In 2001, she became a Researcher with the $\mathrm{Na}$ tional Council for Scientific Research (CONICET), Buenos Aires, Argentina. In 1998 and 1999, she was a Visiting Scholar with the Electromagnetic Communication Research Laboratory, Pennsylvania State University. In February 2002, she was a Visiting Scholar with the Electric and Electronic Engineering Department, University of Colorado at Boulder. She has authored or coauthored over 40 papers in international journals such as Physical Review E, Physical Review Letters, Journal of the Optical Society of America A, Journal of Optics A: Pure and Applied Optics, Optics Communications, Journal of Modern Optics, the IEEE TRANSACTIONS ON ANTENNAS AND PROPAGATION, Nanotechnology, and Optik. She has also authored or coauthored numerous papers in international conferences (URSI-GA, MMET, IEEE Antennas and Propagation Society (IEEE AP-S) International Symposium, JINA International Symposium on Antennas, PIERS, etc.). Since 2002, she has been a Reviewer for numerous journals. She is currently a Topical Editor for Optics Letters. Her main research interest includes enhanced transmission, metamaterials, photonic crystals, scattering from periodic and nonperiodic rough surfaces, finite gratings, modal methods, resonances, superdirectivity, and diffraction.

Dr. Skigin was the recipeint of the Young Scientist Award for the XXIV General Assembly of URSI in 1993 and 1996 and the Young Scientist Prize of the Fifth International Conference on Mathematical Methods for Electromagnetic Theory (MMET*94) in 1994. She was also the recipient of the 1998 Raj Mittra Travel Grant to attend the IEEE AP-S International Symposium and USNC/URSI National Radio Science Meeting in 1998, the ICO Fellowship to attend the XIX International Commission for Optics Conference in 2002, and a research grant to attend the Metamaterials International Conference in 2007. 